VOL. 29 (1984), 13-18.

\title{
SHARP ESTIMATES OF APPROXIMATION BY SOME POSITIVE LINEAR OPERATORS
}

\author{
Ashok Sahai and Govind Prasad
}

Recently, Varshney and Singh [Rend. Mat. (6) 2 (1982), 219-225] have given sharper quantitative estimates of convergence for Bernstein polynomials, Szasz and Meyer-Konig-Zeller operators. We have achieved improvement over these estimates by taking moments of higher order. For example, in case of the MeyerKonig-Zeller operator, they gave the following estimate

$$
\left\|L_{n}(f)-f\right\| \leq\left[\frac{2}{3 \sqrt{3}}+\frac{2}{27}\right]\left(\frac{1}{\sqrt{n}}\right) \omega\left(f^{\prime} ; \frac{1}{\sqrt{n}}\right)
$$

wherein $\|\cdot\|$ stands for sup norm. We have improved this result to

$$
\left\|L_{n}(f)-f\right\| \leq\left[\frac{2}{3 \sqrt{3}}+\left(\frac{105}{8}\right)\left(\frac{4}{27}\right)^{4}\right]\left(\frac{1}{\sqrt{n}}\right) \omega\left(f^{\prime} ; \frac{1}{\sqrt{n}}\right) .
$$

We may remark here that for this modulus of continuity $\omega\left(f^{\prime} ; 1 / \sqrt{n}\right)$ our result cannot be sharpened further by taking higher order moments.

\section{Introduction}

Varshney and Singh [2] have proved the following theorem.

Received 30 August 1983.

Copyright Clearance Centre, Inc. Serial-fee code: 0004-9727/84 $\$ A 2.00+0.00$. 
THEOREM. Let $-\infty<a<b<\infty, p$ be a fixed positive integer. Let $K_{n}$ be a sequence of positive numbers and $\left\{L_{n}\right\}_{n=1}^{\infty}$ be a sequence of positive linear operators, all having the same domain $D$ which contains the restrictions of $1, t, t^{2}, \ldots, t^{2 p}$ to $[a, b]$. Suppose that $\left\{L_{n}(1)\right\}$ is bounded. Let $f^{\prime} \in D$ be continuous in $[a, b]$ with modulus of continuity $\omega\left(f^{\prime} ; \cdot\right)$. Then, for $n=1,2, \ldots$,

(1.1) $\left\|L_{n}(f)-f\right\| \leq\|f\| \cdot\left\|L_{n}(1)-1\right\|+\left\|f^{\prime}\right\| \cdot \mu_{n}^{(1)} \cdot\left\|L_{n}(1)\right\|^{\frac{1}{2}}$

$$
+\omega\left(f^{\prime} ; K_{n} \mu_{1}^{(1)}\right)\left\{\mu_{n}^{(1)}\left\|L_{n}(1)\right\|^{\frac{1}{2}}+\left(\left(\mu_{n}^{(p)}\right)^{2} / 2 p \cdot\left(K_{n} \mu_{n}^{(1)}\right)^{2 p-1}\right)\right\}
$$

where $\mu_{n}^{(r)}=\left\|L_{n}(t-x)^{2 x}(x)\right\|^{\frac{1}{2}}$ for $r=2, p$ and $\|\cdot\|$ norm being sup norm over $[a, b]$.

However if in addition $L_{n}(1)(x)=1$ and $L_{n}(t)(x)=x$, then

(1.2) $\left\|L_{n}(f)-f\right\| \leq \omega\left(f^{\prime} ; K_{n} \mu_{n}^{(1)}\right)\left\{\mu_{n}^{(1)}+\left(\left(\mu_{n}^{(p)}\right)^{2} / 2 p \cdot\left(K_{n} \mu_{n}^{(1)}\right)^{2 p-1}\right)\right\}$.

Using the above results the best possible quantitative estimates of convergence for Bernstein polynomials, Szász and Meyer-Konig-Zeller operators are achieved in the following sections.

\section{Bernstein polynomials}

For $f \in C[0,1]$, let the Bernstein operator of order $n$ be

$$
L_{n}(f)(x)=\sum_{k=0}^{n} B_{n, k}(x) f(k / n)
$$

where

$$
B_{n, k}(x)=\left(\begin{array}{l}
n \\
k
\end{array}\right) x^{k}(1-x)^{n-k}
$$

We prove the following lemma.

LEMMA. Let the function $T_{n, m}$ be defined on $[0,1]$ for positive integers $m$ and $n$ by 


$$
T_{n, m}(x)=\sum_{k=0}^{n} B_{n, k}(x)(k / n-x)^{m}
$$

then

$$
n T_{n, m+1}(x)=x \cdot(1-x) \cdot\left(m T_{n, m-1}(x)+T_{n, m}^{\prime}(x)\right)
$$

Proof. We have

$$
T_{n, m}^{\prime}(x)=\sum_{k=0}^{n} B_{n, k}^{\prime}(x)(k / n-x)^{m}-m T_{n, m-1}(x) .
$$

Because $x \cdot(1-x) \cdot B_{n, k}^{\prime}(x)=B_{n, k}(x) \cdot(k-n x)$ for all $0<k<n$, we write

$$
\begin{aligned}
x \cdot(1-x) \cdot T_{n, m}^{\prime}(x) & \\
& =\sum_{k=0}^{n} x \cdot(1-x) B_{n, k}^{\prime}(x) \cdot(k / n-x)^{m}-m \cdot x \cdot(1-x) \cdot T_{n, m-1}(x) \\
& =\sum_{k=0}^{n} B_{n, k}(x) \cdot n \cdot(k / n-x)^{m+1}-m \cdot x \cdot(1-x) \cdot T_{n, m-1}(x) \\
& =n T_{n, m+1}(x)-m \cdot x \cdot(1-x) T_{n, m-1}(x) .
\end{aligned}
$$

This leads to $(2.1)$.

Using the above result we have

$$
\mu_{n}^{(1)}=1 /(2 \cdot \sqrt{n}), \mu_{n}^{(2)} \doteq(3)^{\frac{1}{2}} / 4 n \text { and } \mu_{n}^{(3)} \doteq\left(15 / 64 n^{3}\right)^{\frac{3}{2}} \text {. }
$$

Choosing $K_{n}=2 n^{-\alpha+\frac{1}{2}}$ for $0<\alpha \leq \frac{1}{2}, p=3$ in (1.2), we obtain, for $f \in C^{1}[0,1]$ and $n \geq 1$,

$$
\left\|L_{n}(f)-f\right\| \leq\left[1+\frac{5}{64 \cdot\left(n^{5\left(\frac{1}{2}-\alpha\right)}\right)}\right] \frac{1}{2 \cdot(\sqrt{n})} \omega\left(f^{\prime} ; n^{-\alpha}\right) .
$$

We note that (2.2) is sharper than the following estimate of Varshney and Singh [2]:

$$
\left\|L_{n}(f)-f\right\| \leq\left[1+\frac{3}{32 \cdot\left(n^{3\left(\frac{1}{2}-\alpha\right)}\right)}\right] \frac{1}{2 \cdot(\sqrt{n})} \omega\left(f^{\prime} ; n^{-\alpha}\right) .
$$




\section{Szász operators}

For $f \in C[0, \infty)$, let the Szász operator of order $n$ be

$$
L_{n}(f)(x)=\sum_{k=0}^{\infty} S_{n, k}(x) f(k / n)
$$

where

$$
S_{n, k}(x)=\exp \cdot(-n x) \cdot(n x)^{k} / k ! .
$$

We now prove the following lemma.

LEMMA. Let the function $T_{n, m}$ be defined on $[0, \infty)$ for positive integers $m$ and $n$ by

$$
T_{n, m}(x)=\sum_{k=0}^{\infty} S_{n, k}(x)(k / n-x)^{m} ;
$$

then

$$
n T_{n, m+1}(x)=x \cdot\left(m T_{n, m-1}(x)+T_{n, m}^{\prime}(x)\right) .
$$

Proof. We have

$$
T_{n, m}^{\prime}(x)=\sum_{k=0}^{\infty} S_{n, k}^{\prime}(x)(k / n-x)^{m}-m T_{n, m-1}(x) .
$$

Now, because $x \cdot S_{n, k}^{\prime}(x)=(k-n x) \cdot S_{n, k}(x)$ for all $0<k<n$, we write

$$
\begin{aligned}
x T_{n, m}^{\prime}(x) & =\sum_{k=0}^{\infty} x \cdot S_{n, k}^{\prime}(x)(k / n-x)^{m}-m \cdot x T_{n, m-1}(x) \\
& =\sum_{k=0}^{\infty} n \cdot S_{n, k}(x)(k / n-x)^{m+1}-m \cdot x T_{n, m-1}(x) \\
& =n T_{n, m+1}(x)-m \cdot x T_{n, m-1}(x) .
\end{aligned}
$$

This leads to (3.1).

Using the above result we have, for $x \in[0, \lambda], 0<\lambda<\infty$,

$$
\mu_{n}^{(1)}=(\lambda / n)^{\frac{1}{2}}, \mu_{n}^{(2)} \doteq(3)^{\frac{1}{2}} \cdot(\lambda / n) \text { and } \mu_{n}^{(3)} \doteq\left(15 \cdot \lambda^{3} / n^{3}\right)^{\frac{1}{2}} \text {. }
$$

Choosing $K_{n}=(1 / \lambda)^{\frac{1}{2}}, p=2$ in (1.2), we obtain, for 
$f \in c^{\perp}[0, \lambda]$ and $n \geq 1$,

$$
\left\|L_{n}(f)-f\right\| \leq\left[\sqrt{\lambda}+\frac{3 \lambda^{2}}{4}\right] \frac{1}{\sqrt{n}} \omega\left(f^{\prime} ; n^{-\frac{1}{2}}\right) .
$$

We note that (3.2) is sharper than the following estimate of Varshney and Singh,

$$
\left\|L_{n}(f)-f\right\| \leq[\sqrt{\lambda}+\lambda / 2] \frac{1}{\sqrt{n}} \omega\left(f^{\prime} ; n^{-\frac{1}{2}}\right),
$$

for all $\lambda<2 / 3$.

Again choosing $K_{n}=(1 / \lambda)^{\frac{1}{2}}, p=3$ in (1.2), we obtain, for $f \in C^{1}[0, \lambda]$ and $n \geq 1$,

$$
\left\|L_{n}(f)-f\right\| \leq\left[\sqrt{\lambda}+5 \lambda^{3} / 2\right](1 / \sqrt{n}) \omega\left(f^{\prime} ; n^{-\frac{1}{2}}\right),
$$

which is sharper than (3.2) for all $\lambda<3 / 10$.

It may be observed that if we have to approximate $f \in C^{\perp}[0, \lambda]$ when $\lambda$ is very small (possibly less than $3 / 10$ ), we may have still sharper estimates of the approximation by using higher values of $p$ in (1.2).

\section{Meyer-Konig-Zeller operators}

For $f \in C[0, \infty)$ let

$$
L_{n}(f)(x)=\sum_{k=0}^{\infty} z_{n, k}(x) f(k /(n+k))
$$

be the Meyer-Konig-Zeller operator of order $n$, where

$$
z_{n, k}(x)=\left(\begin{array}{c}
n+k \\
n
\end{array}\right)(1-x)^{n+1} \cdot x^{k}
$$

We note the following result of Rathore $[1,(2.4), \mathrm{p} .213]$ :

$$
L_{n}\left(|t-x|^{\alpha} ; x\right) \doteq\left(\Gamma(\alpha+1) / 2 / \Gamma\left(\frac{1}{2}\right)\right) \cdot\left(2 x \cdot(1-x)^{2} / n\right)^{\alpha / 2} .
$$

Using (4.1) we have

$$
\mu_{n}^{(1)} \doteq(2 / 3 \cdot \sqrt{3} \cdot \sqrt{n}), \mu_{n}^{(2)} \doteq(\sqrt{3} \cdot 4 / 27 n), \mu_{n}^{(3)} \doteq\left(\sqrt{5} \cdot 8 / 81 \cdot n^{3 / 2}\right)
$$


and

$$
\mu_{n}^{(4)} \doteq\left(\sqrt{105} \cdot 16 / 729 n^{2}\right)
$$

Choosing $k_{n} \doteq 3 \sqrt{3} / 2, p=4$ in (1.2), we obtain, for $f \in C^{\lambda}[0,1)$ and $n \geq 1$,

(4.2) $\quad\left\|L_{n}(f)-f\right\| \leq\left[2 / 3 \sqrt{3}+(105 / 8) \cdot(4 / 27)^{4}\right](1 / \sqrt{n}) \omega\left(f^{\prime} ; 1 / \sqrt{n}\right)$.

We note that this is sharper than the following estimate given by varshney and Singh [2]:

$$
\left\|L_{n}(f)-f\right\| \leq[2 / 3 \sqrt{3}+2 / 27](1 / \sqrt{n}) \omega\left(f^{\prime} ; 1 / \sqrt{n}\right) .
$$

We may remark that the estimate in (4.2) cannot be bettered by taking higher value of $p$ in (1.2) for modulus of continuity $\omega\left(f^{\prime} ; 1 / \sqrt{n}\right)$.

\section{References}

[1] R.K.S. Rathore, "Lipschitz-Nikolskil constants and asymptotic simultaneous approximation of the $M_{n}$-operators", Aequationes Math. 18 (1978), 206-217.

[2] Om P. Varshney and S.P. Singh, "On degree of approximation by positive linear operators", Rend. Mat. (7) 2 (1982), 219-225.

Department of Mathematics,

University of Roorkee,

Roorkee,

U.P. 247667,

India. 